\title{
Uterine Reconstruction Due to a Giant Myoma. A Case Report and Literature Review
}

\author{
M. N. Gennaro Della Rossa, B. Navarro Santana, M. Rodríguez Verdejo-Arias, \\ E. Valer Cardenas, J. Plaza Arranz \\ Department of Gynecology and Obstetrics, “Fundación Jiménez Díaz” University Hospital, Madrid, Spain \\ Email: nataliagennaro@hotmail.com
}

Received 14 October 2015; accepted 22 January 2016; published 25 January 2016

Copyright (C) 2016 by authors and Scientific Research Publishing Inc.

This work is licensed under the Creative Commons Attribution International License (CC BY). http://creativecommons.org/licenses/by/4.0/

\section{(c) (i) Open Access}

\begin{abstract}
Giant myomas, defined as those $>9 \mathrm{~cm}$, are uncommon. Due to the difficulties caused by its enormous size, the usual surgical treatment is a simple total hysterectomy. The surgery may be performed by either laparoscopy or laparotomy. However, the case presents a 45-year-old woman who was diagnosed with a giant myoma of $23 \mathrm{~cm}$. The patient is asymptomatic and does not accept the indication of a simple total hysterectomy because of her fear to lose her femininity and a feeling of mutilation and unfulfilled genesic desire. A personalized procedure was performed: myomectomy by laparotomy with subsequent uterine reconstruction. The case is described to focus the interest on giant uterine myomas, taking into account the limited availability of literature on the topic, and to highlight the reconstruction after myomectomy.
\end{abstract}

\section{Keywords}

Giant Myoma, Myomectomy, Uterine Reconstruction

\section{Introduction}

Fibroids are the most frequent benign tumors in women. The finding of such tumor is considered extraordinary before menarche and the decrease after menopause is normal. Its pathogenesis is closely related to sex hormones, particularly estrogen, genetic predisposition and growth factors [1]. Maximum incidence is observed in women between 30 and 50 years and it is present in up to $40 \%$ of women. The prevalence of the tumor during pregnancy is $10 \%$ and it is the cause of infertility in $2 \%-3 \%$ cases in which the active management is recommended to improve reproductive rate [2]. The location and size can vary, the size can range from a few millimeters to sev- 
eral centimeters and a giant myoma is considered when it exceeds a size of $9 \mathrm{~cm}$ or a weight of $800 \mathrm{gr}$ [3]. Its prevalence is very difficult to define and it is estimated between $4 \%$ and $25 \%$ of cases [3] [4].

Fibroids are often asymptomatic but $30 \%$ of the patients affected by fibroids present heavy menstrual flow or other alterations such as pelvic discomfort, menometrorrhagia, dyspareunia, urinary disorders, back pain or infertility [2] [4]. Symptomatic fibroids require treatment, being hysterectomy by laparotomy or laparoscopy of the classic option. This condition is responsible for $60 \%$ of hysterectomies performed in Western countries. Another possible treatment is surgical myomectomy by laparotomy, laparoscopy or hysteroscopy. The kind of procedure depends on the location, number and size of the fibroids. It is also possible to administer a preoperative treatment in order to reduce the volume of the myoma and the uterine bleeding with gonadotropin-releasing hormone agonist or, preferably, Ulipristal Acetate. Non-surgical options such as: embolization selective trans-arterial and therapy HIFU (High Intensity Focused Ultrasound) [2]-[4] are added to the classic surgical treatments.

In the case of giant fibroids, scientific evidence on prescribed treatment is limited or null in terms of conservative techniques. The size increase often makes it difficult to perform the usual surgical techniques and a total or even a subtotal hysterectomy is indicated. The literature is limited to the technique of excision of giant uterine fibroids and reconstruction, but there are cases in which the correct indication could be the following:

These cases are summarized in 2 options:

- Patient with unfulfilled genesic desire.

- Patients who do not want a hysterectomy.

It is important to remember that all reductive interventions could trigger an emotional disorder whose intensity depends not only on the factors relating to the organ or disease in question but also on the impact that the feeling of loss of personal integrity has, as well as the sexual and reproductive consequences. Among these interventions in women, hysterectomy is seen as a mutilating surgery and can exercise greater impact than any other intervention. The Health and Gender Report Ministry of Health of Spain [5] emphasizes on how surgical procedures such as hysterectomy or mastectomy appear as a source of vulnerability and require a redefinition of femininity when it has been supported and symbolized in these organs.

This case report includes a review of the literature on myomectomy and the different techniques of uterine reconstruction which allow patients to keep their uterus, the unfulfilled genesic desire and the full-body image, thus providing a better quality of life.

\section{Clinical Case}

White 45-year-old patient. Medical history: anxiety depressive disorder with 4 years of evolution. Surgical history: saphenectomy in the right lower limb in 2010. She had no relevant family history. No toxic habits. Gyneco-Obstetric history included one gestation with a voluntary interruption when the patient was 32 years old.

Gynecological examination: a mass occupying the whole abdomen above $3 \mathrm{~cm}$ supra-umbilical was discovered. Abdominal ultrasound showed uterus with intramural fibroid $167 \mathrm{~mm} \times 160 \mathrm{~mm} \times 230 \mathrm{~mm}$. Magnetic Resonance Imaging (MRI) of pelvic area displayed an intramural uterine mass $230 \mathrm{~mm} \times 110 \mathrm{~mm} \times 190 \mathrm{~mm}$. T1 sequences were observed as a predominantly hypo-intense and homogenous solid mass and T2 sequences had heterogeneous and hyper-intense signal areas inside related to cystic necrotic areas, in keeping with giant fibroid type 4 according to the FIGO classification. The anterior myometrium had a maximum thickness of $20 \mathrm{~mm}$ and the posterior face had a maximum thickness $5 \mathrm{~mm}$. The endometrium was located above the fibroid with a maximum fibroid interface of $3 \mathrm{~mm}$. Adnexal structures were not identified. There was no dilatation of the urinary tract. Hemoglobin was $11.9 \mathrm{~g} / \mathrm{dL}$ (Figure 1).

Initially, a laparotomy/laparoscopic total hysterectomy was proposed. The patient sought to preserve her uterus due to feelings of mutilation associated with hysterectomy and the loss of femininity associated with the incompleteness of her internal genitals. She also understood the few chances of pregnancy due to old age and the possibility of requiring future treatments for new uterine disorders.

Lastly, the surgery proposed was myomectomy with uterine reconstruction, intra-operative biopsy by laparotomy. The corresponding preoperative consent was signed. Surgical risks ASA II. Two units of red blood cells were reserved.

Midline laparotomy with a lengthwise opening of $12 \mathrm{~cm}$ of the abdominal wall was performed. The enlarged uterus is outsourced, protected by moist compresses. The upper structures of the uterine pedicle were individualized. Both round ligaments and adnexal (tubes and ovaries) were found macroscopically normal. Wet pad was placed to insulate and protect intestines. 


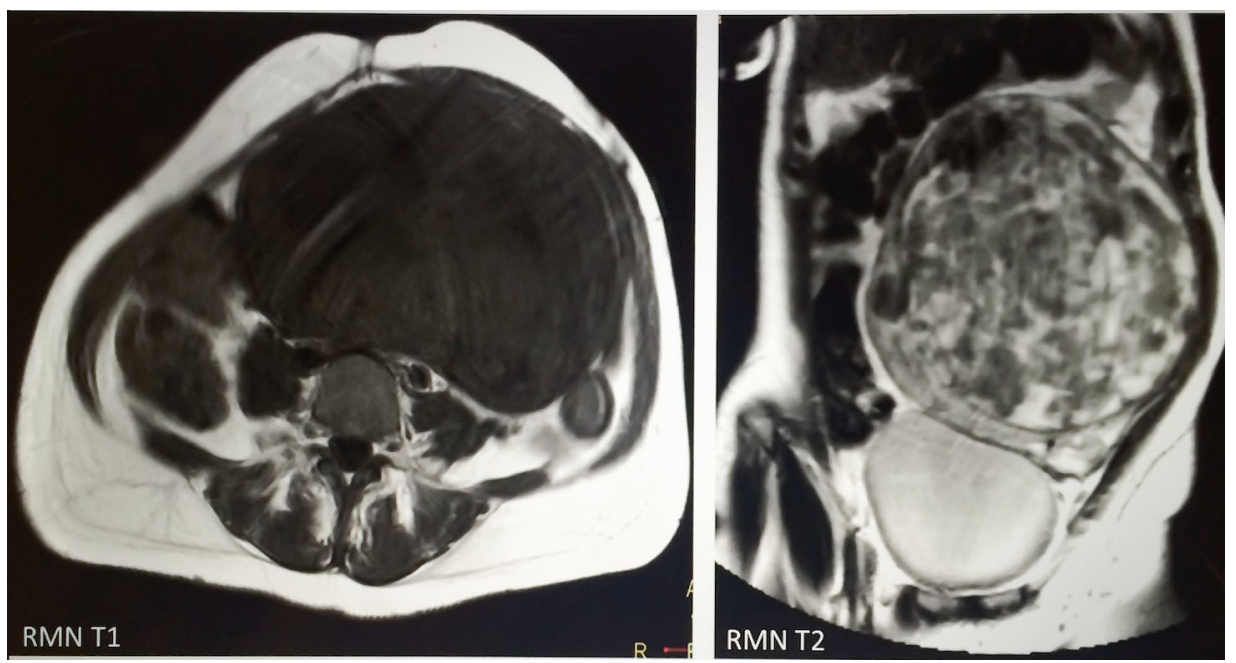

Figure 1. Image of MRI in T1 and T2 respectively, showing giant uterine fibroid compatible mass.

$1^{\text {st }}$ Time: MYOMECTOMY. To reduce bleeding during myomectomy, a peri-cervical Foley catheter was placed and tied to decrease uterine blood flow [6]. From that moment, the ischemia time was controlled and a limit of 30 minutes was accepted. Then, a longitudinal incision was made in the midline of the posterior face and fundic uterus at about $7 \mathrm{~cm}$. This approach was decided due to the thinning of the rear wall with a maximum thickness of $5 \mathrm{~mm}$, contrary to the anterior face thickness of $2 \mathrm{~cm}$ and the impossibility of subsequent myometrium retraction after myomectomy. Optimal dissection plane (fibroid capsule) was individualized and myomectomy was performed according to the usual traction-countertraction technique. With complete enucleation of the myoma and after ligation of the vascular pedicle, the piece $(230 \times 190 \times 120 \mathrm{~mm})$ was extracted and intra-operative biopsy was performed. The biopsy was reported as: uterine leiomioma with minimal nuclear atypia and low mitotic activity with interstitial edematous degeneration without signs of necrosis.

$2^{\text {nd }}$ Time: UTERINE RECONSTRUCTION. Ischemia time was 17 minutes. The endometrial cavity was opened corresponding to the uterine fundus and $2 \mathrm{~cm}$ were sutured with continuous suture 2/0 vicryl. Next, a metroplasty was performed which included a rhomboid resection from the posterior uterine face, due to its extreme thinning and no retraction of uterine tissue before beginning this surgery. The rhomboid resection included two top faces of $4.5 \mathrm{~cm}$ and two of $2 \mathrm{~cm}$ from the underside. Myometrium of the upper faces, in one discontinuous layer of 0/0 vicryl sutured (due to the extreme thinness of the myometrium, which did not allow the suture in two layers as a choice) being the midline suture of uterus in inverted $\mathrm{T}$. The lower sides were sutured with the over-flap technique (overlapping myometrium) with interrupted sutures with $0 / 0$ vicryl, in order to avoid an excessive reduction of the uterine fundus, and increase the strength of the scar to an eventual pregnancy.

After that, the tourniquet was removed, peri-cervicaly serving a maximum time of 27 minutes [7]. Subsequently, a Foley catheter was introduced in the uterus and was inflated with $50 \mathrm{cc}$ of a saline solution to prevent the collapse of the uterine walls and eventual adhesions. No active bleeding was found. Finally, a layer of oxidized regenerated cellulose covered the entire myomectomy scar to prevent adhesions. Drain pack in Douglas space for monitoring possible postoperative bleeding had been left and the abdominal wall was closed using continuous suture with $0 / 0$ vicryl in fascia, then subcutaneous tissue approximation and umbilical reconstruction and intradermal suture leather with 4/0 polysorb was performed. The bleeding accounted was $230 \mathrm{cc}$ of blood.

The immediate postoperative recovery had not complications. At 24 hours the patient had a hemoglobin control of $10.9 \mathrm{mg} / \mathrm{dl}$. Drainage pack with 50 cc of debit and intrauterine Foley catheter were removed. Three days after surgery, with clinical and hemodynamic stability, a uterine ultrasound control with no bruises and oral iron treatment was indicated and the patient went home.

First postoperative control: 10 days after surgery: the patient had little genitoragia. Gynecological ultrasound: uterus AVF of $80 \times 70 \times 70 \mathrm{~mm}$, with an undefined endometrial line and normal ovaries. Absence of free fluid in Douglas.

Second postoperative control: 30 days after surgery: minor genitorragia. Hemoglobin was $11.1 \mathrm{mg} / \mathrm{dl}$. Gyne- 
cological trans-vaginal ultrasound: Uterus in AVF of $70 \times 60 \times 40 \mathrm{~mm}$, lineal endometrium and normal ovaries. There was absence of free fluid in Douglas.

Third postoperative control: four months after surgery the patient was satisfied and happy by the preservation of her uterus. Regular periods had started again. Other results: control hemoglobin of $11.9 \mathrm{mg} / \mathrm{dl}$; hormonal profile on $3^{\text {rd }}$ day of period FSH $-14 \mathrm{mIU} / \mathrm{ml}$; LH $6 \mathrm{mIU} / \mathrm{ml}$ and estradiol $40 \mathrm{mIU} / \mathrm{ml}$. Hystero-sonography was performed yielding the following results: normal uterine morphology, inter-cornual distance of $57 \mathrm{~mm}$ and both fallopian tubes were permeable (Figure 2).

Seeing this favorable progress, it was decided start annual gynecological follow-ups.

\section{Discussion}

Giant fibroids, defined as those larger than $9 \mathrm{~cm}$, are not frequent and have a prevalence [3] of 25\% in white women [4], according to some authors; while $50 \%$ has been reported in black women [3]. However, an exact prevalence is difficult to determine. The symptoms include a wide range of possibilities such as [8]: menometrorrhagia, pelvic pressure, urinary symptoms due to compression of urethra with hydro-nephrosis [9] or bladder compression with symptoms of urgency and urge incontinence [10], edema, or pelvic varices MMII, thrombosis or compression symptoms sacral plexus. Other symptoms described included unusual pulmonary hypertension, respiratory failure [11], pseudo-Meigs syndrome [12], difficulty to walk, tiredness [13] and arthrogryposis in pregnancy [14].

The FIGO classification according to their location is [15]:

- Submucosal fibroids:

Type 0: Submucous $100 \%$

Type 1: Intramural $<50 \%$

Type 2: Intramural $>50 \%$

- Other fibroids:

Type 3: Contacts the endometrium. 100\% intramural

Type 4: Intramural

Type 5: Subserous $>50 \%$ intramural

Type 6: Subserous $<50 \%$ intramural

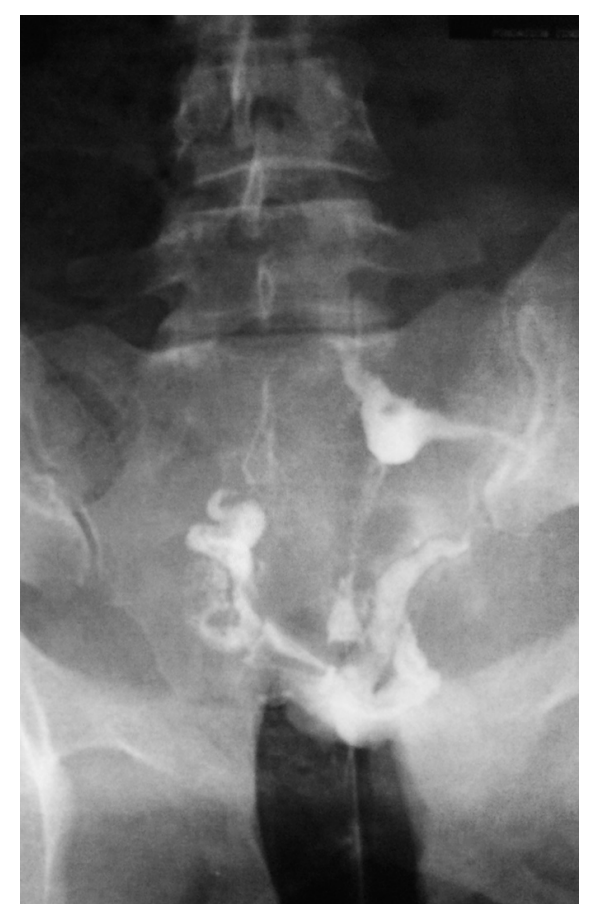

Figure 2. Radiographic image hysterosonography achieved by demonstrating the anatomy of the endometrial cavity and both permeability after surgery for uterine reconstruction uterine tubes. 
Type 7: Subserosal peduncled

Type 8: Other (cervical, parasitic, broad ligament)

- Hybrid fibroids (impacts on myometrium and serosa)

There are reports of fibroids found in the retroperitoneal space [16], having to make the differential diagnosis with other masses found in the same area, which sometimes can be malignant, for example: the mucinous cystadenoma, cystic lymphangioma, cystic mesothelioma and Mullerian cyst. In other cases, the tumors are benign such as urinomas and lymphoceles. Myomas have also been described in Retzius space [10].

Gynecological trans-vaginal ultrasound is the technique of choice for the diagnosis because it is the most cost-effective with perfect display [6]. If the fibroid exceeds the pelvis, abdominal ultrasound is required to view the complete mass. However, MRI is considered more accurate for the diagnosis and if the fibroid is too large, an abdominal and pelvic MRI [1] will also be required for its evaluation.

In most cases, the treatment of giant myomas is surgical [8], that is, hysterectomy by laparotomy [3] or laparoscopy vs. myomectomy by laparotomy, as in our case, or by laparoscopy [17].

Hysterectomy is indicated in the following cases:

- Acute bleeding unresponsive to other therapies [18].

- Unfulfilled genesic desire and future risk of developing: adenomyosis, endometrial hyperplasia and premalignant lesions or tumors of the cervix, endometrium or ovary.

- Unfulfilled genesic desire with multiples fibroids or significant symptoms.

- If the patient wishes so.

Regarded as benefit of hysterectomy:

- To remove current symptoms and probably future symptoms which can occur in relation to other pathologies or uterine fibroids.

Disadvantages of hysterectomy:

- Increase in morbidity that would include damage to other organs.

Myomectomy would be indicated in the following cases:

- Unfulfilled genesic desire, if reproductive capacity can be improved.

- If the patients wish to keep their uterus (as in the case described).

Advantage of myomectomy:

- Improves symptoms and preserves uterus.

Disadvantages of myomectomy:

- New fibroids may occur from new clones of abnormal myocytes.

- Intra-operative bleeding

Before surgery, it would be appropriate to correct preoperative anemia with iron, EPO or both, depending on whether the anemia is ferropenic, chronic or mixed, respectively; thus avoiding eventual blood transfusions because they are not exempt from risks with increased morbidity and mortality [19]. Also during myomectomy, it is necessary to perform different procedures to prevent bleeding. Currently, there is moderate quality evidence that misoprostol can reduce bleeding during myomectomy, while there is lower evidence that bupivacaine plus epinephrine, tranexamic acid, the gelatin-thrombin matrix, a tourniquet peri-cervical, ascorbic acid, dinoprostone, ligation in handle and a fibrin sealant patch can reduce bleeding during myomectomy. Nevertheless, there is no evidence that oxytocin, morcellation and temporary clamping of the uterine artery can reduce blood loss.

- Another disadvantage of myomectomy that should be taken into account is the uterine rupture in a subsequent pregnancy.

The incidence of uterine rupture is $1 \%$ according to Dubuisson et al. [20]. It is most common during the third trimester of pregnancy. However there have been reports of uterine ruptures during the second trimester [21]. In addition, laparotomy is also more commonly used in the myomectomy, especially if it is performed by an inexperienced surgeon, considering the extension of myometrial tissue destroyed if there is a frequent use of electrocoagulation and if the myometrium is sutured with a single layer [22].

Uterine reconstruction is required in cases of:

- Giant myoma: the myometrial tissue remains very thin after the giant myoma enucleation and does not preserve it contractile activity. The following techniques are chosen: inverted suture (a), intussusception of the myometrium (b), overlapping of the remaining myometrial tissue (c), and resection of remnant tissue (d) [23] (Figures 3(A)-(D)).

- Multiple fundal fibroids: Riedberg technique (e) [23] (Figure 3(F)). 


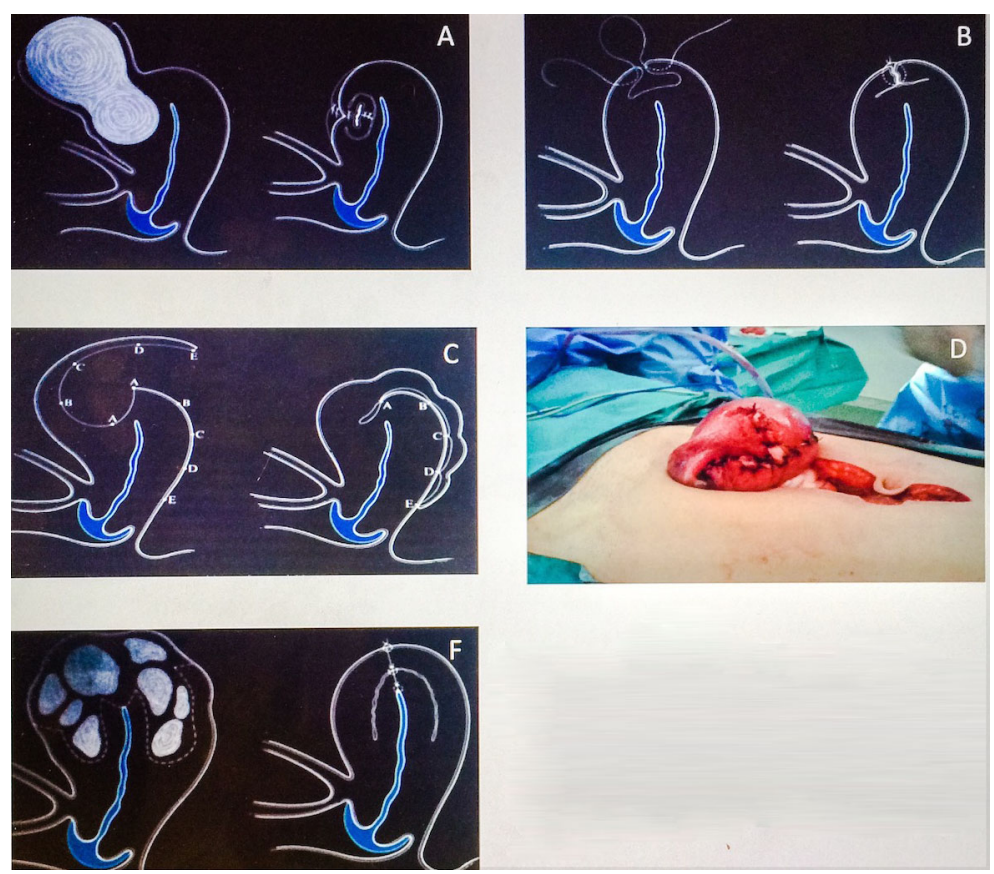

Figure 3. (A) Inverted (a) suture. Playing Hirsch, O. Käser, Iklé FA Gynecological Surgery Atlas of breast surgery, urological surgery and gastrointestinal surgery. Madrid. Marban. 2003. 153-163; (B) Suture of myometrial tissue ingrowth (b). Playing Hirsch, O. Käser, Iklé FA Gynecological Surgery Atlas of breast surgery, urological surgery and gastrointestinal surgery. Madrid. Marban. 2003. 153-163; (C) Suture myometrial tissue overlap the remaining (c). Playing Hirsch, O. Käser, Iklé FA Gynecological Surgery Atlas of breast surgery, urological surgery and gastrointestinal surgery. Madrid. Marban. 2003. 153-163; (D) Suture T Reversed. With acontractile cut tissue and overlay flap over; (F) Technique Friedberg (e). Playing Hirsch, O. Käser, Iklé FA Gynecological Surgery Atlas of breast surgery, urological surgery and gastrointestinal surgery. Madrid. Marban. 2003. 153-163.

An excellent haemostatic suture should be made because it is the main factor to prevent adhesions after myomectomy, but there are some studies in which the use on the surgical bed of absorbable materials such as oxidized regenerated cellulose or non-absorbable materials like polytetrafluoroethylene have reduced adhesions between uterus and other pelvic or abdominal organs [24] [25].

Considering that bleeding after a giant myomectomy is possible, a drain may be left in the Douglas space. The uterus is usually enlarged at the end of the surgery, but its size is reduced after several weeks or months [23].

It is worth emphasizing that in our case it was decided to perform surgery (myomectomy) due to the oversize rapid growth of the fibroid. However, it seems that fibroids which grow rapidly and are asymptomatic, and the diagnostic imaging is consistent with fibroids, the best thing would be its observation because the sarcoma prevalence is very low [26]; nevertheless, the literature does not mention whether there is a maximum size to allow the observation and not the intervention.

It was decided to perform an intra-operative biopsy to rule out a malignant diagnosis. However, the hypothesis that sarcomas develop from fibroids has not been proved [26]. Nevertheless, if the malignization of a myoma occurs, it would be an exceptional event [27]-[29].

Our patient is trying to get pregnant, without success yet. The literature has reported cases of gestation after myomectomy of a giant fibroid [8]. The best way of delivery would be a caesarean section (before the onset of prodromal labor).

Above all, our patient is satisfied and very pleased with the results. The literature has extensive reports on this topic. One of them was written by Mackinger, Graf, Keck et al. [30] who studied a group of women who had undergone hysterectomy and revealed significantly higher levels of health complaints like depression, and diverse sexual and psychological disorders, compared to the group which had not undergone hysterectomy, considering that in the hysterectomy group, the patients showed psychological distress previous surgery. Rannestad [31] showed how hysterectomy does not cause adverse psychological results in women without psychological morbidity previous surgery, being the psychological status before surgery the best predictor of subsequent psy- 
chopathologies. Thus, women diagnosed with a major depressive disorder before surgery have higher probability of continuing with this diagnosis post-intervention or even a worsening of symptoms. Thus, preoperative psychopathology is a predictor of postoperative psychopathology [31].

\section{Conclusions}

Fibroids can achieve huge sizes as indicated by some reports: Hunt in 1888 found a fibroid which weighed 63.6 $\mathrm{kg}$ in an autopsy; Behrend in 1930 removed a uterine fibroid of $60.7 \mathrm{~kg}$; Singhabhandhu in 1973 removed the largest tumor so far $(45.5 \mathrm{~kg})$ from a patient who survived the intervention. Oelsner reported 2 cases in May 2003, one of $40 \mathrm{~kg}$ and another of $43 \mathrm{~kg}$. More recently, Costa reported the removal a $9.5 \mathrm{~kg}$ fibroid.

Although uncommon, whenever these tumors are present they constitute a challenge for gynecologists. A hysterectomy is the most standard treatment in these cases, but we want to ensure that a myomectomy can performed, even by laparoscopy, including uterine reconstruction to preserve the woman's uterus and ovarian functions or to prevent great moral suffering.

\section{Recommendation}

We recommend the following guidelines prior to myomectomy:

- Evaluation of unfulfilled genesic desire and the meaning of the proposed surgery in patients, taking into account previous psychological disorders, which may worsen after surgery if they are not handled properly.

- Evaluation of symptoms, considering also uncommon symptoms of fibroids.

- MRI assessment of the location of the myoma and alterations in the anatomy of the myometrium.

- Preoperative signing of complete informed consent.

- Correction of preoperative anemia.

- Reserve of blood backup.

- Determine the need of ureteral catheterization.

- Determine the need of bowel preparation.

- Prepare adequate antithrombotic guidelines.

- Define laparotomy or laparoscopic approach.

- Perform maneuvers or techniques to reduce uterine bleeding.

- Define the uterine incision to be performed taking into account the location of the myoma, the anatomical changes and the uterine circulation, the front face is preferred.

- An intra-operative biopsy can be performed, though it is not indispensable.

- Perform reconstruction if the myometrium does not retract or contract properly, although it is important to bear in mind that the uterus will be slightly larger than desired and will eventually retract.

- Make correct hemostatic sutures. If possible the sutured must be performed in two layers.

- Use maneuvers to prevent intrauterine and extra uterine adhesions.

- Perform early control for potential bleeding or bruising.

- Evaluate postoperative uterine morphology by means of gynecological trans-vaginal ultrasound and request historiography to assess tubal permeability if it is required.

\section{Conflicts of Interest}

The authors declare that there is no actual or potential conflict of interest in relation to this case.

\section{References}

[1] Calaf, J., Arqué, M., Porta, O. and D’Angelo, E. (2013) The Fibroid as Clinical Problem. Medicina Clínica, 141, 1-6. http://dx.doi.org/10.1016/S0025-7753(13)70045-9

[2] Mara, M., Maskova, J., Fučíkova, Z., Kuzel, D., Belsan, T. and Sosna, O. (2008) Midterm Clinical and First Reproductive Results of a Randomized Controlled Trial Comparing Uterine Fibroid Embolization and Myomectomy. CardioVascular and Interventional Radiology, 31, 73-85. http://dx.doi.org/10.1007/s00270-007-9195-2

[3] Santana Pedraza, T., Estepa Perez, J.L. and Enteza, M.J.F. (2013) Giant Uterine Fibromyoma. Report of a Case. Medisur, 11.

[4] García, V., Cernadas, S. and Buján, V. (2012) The All Symptomatic Fibroids. Myomectomy or Uterine Artery Embo- 
lization. Revista Chilena de Obstetrica Y Ginecologia, 77, 255-258.

[5] Ministry of Health of Spain (2006) The Central Ages of Life. Health and Gender Report 2006.

[6] Kongnyuy, E.J. and Wiysonge, C. (2014) Interventions to Reduce Haemorrhage during Myomectomy for the Treatment of Fibroids (Cochrane Review). Cochrane Database of Systematic Reviews, 8, Article No. CD005355. http://dx.doi.org/10.1002/14651858.CD005355.pub5

[7] Breech, L.L. and Rock, J.A. (2011) Te Linde Surgical Gynecology. In: Rock, J., Ed., Myomectomy and Uterine Leiomyomas. You Linde Surgical Gynecology, Editorial Medica Panamericana, 10th Edition, 687-726. [Spain]

[8] Ezugwu, E.C., Iyoke, C.A., Ezugwu, F.O. and Ugwu, G. (2014) Successful Pregnancy Following Myomectomy for Uterine Fibroid Giant in an Infertile Woman. Journal of Reproduction \& Infertility, 15, 233-236.

[9] Semczuk, A., Skorupski, P., Olcha, P., Skomra, D., Rechberger, T. and Gogacz, M. (2009) Giant Uterine Leiomyomas Bilateral Hydronephrosis Causing with Coexisting in Polyp Endometrial Cancer: A Case Study. European Journal of Gynaecological Oncology, 30, 344-346.

[10] Pepe, F., Pepe, P., Rapisarda, F., Fauzia, M. and Giunta, M. (2013) Giant Leiomyoma of the Retzius Space: A Case Report. Case Reports in Obstetrics and Gynecology, 2013, 371417. http://dx.doi.org/10.1155/2013/371417

[11] Oelsner, G., Elizur, S.E., Frenkel, Y. and Carp, H. (2003) Giant Uterine Tumors: With Cases Two Different Clinical Presentations. Obstetrics \& Gynecology, 101, 1088-1091. http://dx.doi.org/10.1016/S0029-7844(02)02621-2

[12] Landrum, L.M., Rutledge, T.L., Osunkoya, A.O. and Mannel, R. (2008) Associated with Uterine Leiomyoma PseudoMeigs Síydrome Mimicking Ovarian Carcinoma. The Journal of the Oklahoma State Medical Association, 101, 38-39.

[13] Nappi, L., Matteo, M., Giardina, S., Rosenberg, P., Indraccolo, U. and Greco, P. (2008) Management of Uterine Myoma Giant. Archives of Gynecology and Obstetrics, 278, 61-63. http://dx.doi.org/10.1007/s00404-007-0523-1

[14] Vila-Vives, J.M., Hidalgo-Mora, J.J., Soler, I., Rubio, J., Quiroga, R. and Perales, A. (2012) Fetal Arthrogriposis Secondary to a Giant Maternal Uterine Leiomyoma. Case Reports in Obstetrics and Gynecology, 2012, Article ID: 726732.

[15] Munro, M.G., Critchley, H.O., Broder, M.S. and Fraser, I.S., FIGO Working Group on Menstrual Disorders (2011) FIGO Classification System (PALM-COEIN) for Causes of Abnormal Uterine Bleeding in Non Gravid Women of Reproductive Age. International Journal of Gynecology \& Obstetrics, 113, 3-13.

[16] Funaki, K., Fukunishi, H., Tsuji, Y., Maeda, T. and Takahashi, T. (2013) Giant Cystic Leiomyoma of the Uterus Occupying the Retroperitoneal Space. Journal of Radiology, 7, 35-40.

[17] Sinha, R., Hegde, A., Warty, N. and Patil, N. (2003) Laparoscopic Excision of Very Large Myomas. Journal of The American Association of Gynecologic Laparoscopists, 10, 461-468. http://dx.doi.org/10.1016/S1074-3804(05)60145-4

[18] Brito, L.G., Magnani, P.S., Azevedo Trapp, A.E. and de Freitas, M.M.S. (2011) Giant Prolapsed Submucous Leiomyoma: A Surgical Challenge for Gynecologists. Clinical and Experimental Obstetrics \& Gynecology, 38, $299-300$.

[19] García, V.M., de Bernabé Sala, M.Á.G., Diana, K.N., Pericas, B.C. and Nebot, A.G. (2013) Anemia Surgical Risk Factor. Medicina Clínica, 141, 47-54. http://dx.doi.org/10.1016/S0025-7753(13)70053-8

[20] Dubuisson, J.B., Fauconnier, A., Deffarges, J.V., Norgaard, C., Kreiker, G. and Chapron, C. (2000) Pregnancy Outcome Following Laparoscopic Myomectoy and Deliveries. Human Reproduction, 5, 869-873. http://dx.doi.org/10.1093/humrep/15.4.869

[21] Kiseli, M., Artas, H., Armagan, F. and Dogan, Z. (2013) Spontaneous Rupture of Uterus in Pregnancy Midtrimester Due to Increased Pressure with Previous Uterine Laparoscopic Myomectomy. International Journal of Fertility and Sterility, 7, 239-242.

[22] Kim, M.S., Uhm, Y.K., Kim, J.Y., Jee, B.C. and Kim, Y.B. (2013) Obstetric Outcomes after Uterine Myomectomy: Laparoscopic versus Laparotomic Approach. Obstetrics \& Gynecology Science, 56, 375-381. http://dx.doi.org/10.5468/ogs.2013.56.6.375

[23] Hirsch, H.A., Käser, O. and Iklé, F.A. (2003) Conservative Surgery of Uterine Fibroids and Uterine Malformations. In: Hirsch, H.A., Käser, O. and Iklé, F.A., Eds., Gynecological Surgery Atlas of Breast Surgery, Urological Surgery and Gastrointestinal Surgery, Marban, Madrid, 153-163.

[24] Diamond, M.P. (1996) Reduction of Adhesions after Uterine Myomectomy by Seprafilm Membrane (HAL-F): A Binded, Prospective, Randomized, Multicenter Clinical Study. Seprafilm Adhesion Study Group. Fertility and Sterility, 66, 904-910.

[25] Mais, V., Ajossa, S., Piras, B., Guerrieiro, S., Marongiu, D. and Melis, G.B. (1995) Prevention of De-Novo Adhesion Formation after Laparoscopic Myomectomy: A Randomized Trial to Evaluate the Effectiveness of an Oxidized Regenerated Cellulose Absorbable Barrier. Human Reproduction, 10, 3133-3135.

[26] Schwartz, P.E. and Kelly, M.G. (2006) Malignant Transformation of Myomas: Myth or Reality? Obstetrics \& Gynecology Clinics of North America, 33, 183-198. http://dx.doi.org/10.1016/j.ogc.2005.12.003 
[27] Yanai, H., Wani, Y., Notohara, K., Takada, S.I. and Yoshino, T. (2010) Uterine Leiomyosarcoma Arising in Leiomyoma: Clinicopathological Study of Four Cases and Literature Review. Pathology International, 60, 506-509. http://dx.doi.org/10.1111/j.1440-1827.2010.02549.x

[28] Rotmensch, J., Bosnyak, S. and Montag, A. (1993) Malignant Transition of Uterine Leiomyomata. International Journal of Gynecology \& Obstetrics, 42, 47-49. http://dx.doi.org/10.1016/0020-7292(93)90449-7

[29] Scurry, J. and Hack, M. (1990) Leiomyosarcoma Arising in a Lipoleiomyoma. Gynecologic Oncology, 39, $381-383$. http://dx.doi.org/10.1016/0090-8258(90)90271-L

[30] Mackinger, H.F., Graf, A.H., Keck, E., Tempfer, C. and Kainz, C. (2001) Differences in the Psychological Status of Hysterectomy and Non-Hysterectomy Women. Wiener klinische Wochenschrift, 113, 954-959.

[31] Rannestad, T. (2005) Hysterectomy: Effects on Quality of Life and Psychological Aspects. Best Practice \& Research Clinical Obstetrics \& Gynaecology, 19, 419-430. http://dx.doi.org/10.1016/j.bpobgyn.2005.01.007 